\title{
Notch Signaling Alters Sensory or Neuronal Cell Fate Specification of Inner Ear Stem Cells
}

\author{
Sang-Jun Jeon, ${ }^{1,2 \star}$ Masato Fujioka, ${ }^{1,2 \star}$ Shi-Chan Kim, ${ }^{1,2}$ and Albert S. B. Edge ${ }^{1,2,3}$ \\ ${ }^{1}$ Department of Otology and Laryngology, Harvard Medical School, Boston, Massachusetts $02115,{ }^{2}$ Eaton-Peabody Laboratory, Massachusetts Eye and Ear \\ Infirmary, Boston, Massachusetts 02114, and ${ }^{3}$ Program in Speech and Hearing Bioscience and Technology, Division of Health Science and Technology, \\ Harvard University and Massachusetts Institute of Technology, Cambridge, Massachusetts 02139
}

Multipotent progenitor cells in the otic placode give rise to the specialized cell types of the inner ear, including neurons, supporting cells, and hair cells. The mechanisms governing acquisition of specific fates by the cells that form the cochleovestibular organs remain poorly characterized. Here we show that whereas blocking Notch signaling with a $\gamma$-secretase inhibitor increased the conversion of inner ear stem cells to hair cells by a mechanism that involved the upregulation of bHLH transcription factor, Math1 (mouse Atoh1), differentiation to a neuronal lineage was increased by expression of the Notch intracellular domain. The shift to a neuronal lineage could be attributed in part to continued cell proliferation in cells that did not undergo sensory cell differentiation due to the high Notch signaling, but also involved upregulation of Ngn1. The Notch intracellular domain influenced Ngn1 indirectly by upregulation of Sox2, a transcription factor expressed in many neural progenitor cells, and directly by an interaction with an RBP-J binding site in the Ngn1 promoter/enhancer. The induction of Ngn1 was blocked partially by mutation of the RBP-J site and nearly completely when the mutation was combined with inhibition of Sox 2 expression. Thus, Notch signaling had a significant role in the fate specification of neurons and hair cells from inner ear stem cells, and decisions about cell fate were mediated in part by a differential effect of combinatorial signaling by Notch and Sox 2 on the expression of bHLH transcription factors.

\section{Introduction}

Self-renewing cells with the ability to give rise to the differentiated cell types of the inner ear, including hair cells, supporting cells, and neurons (Oshima et al., 2007; Martinez-Monedero et al., 2008), have been isolated from the inner ear by neurosphere formation (Li et al., 2003a; Oshima et al., 2007). Mechanisms for determining the fate of these cell types from early progenitors are essential for establishing a functional sensory epithelium in the vestibular and auditory organs. The progenitors were shown to be present in the vestibular organs throughout life and in the cochlea for the first few postnatal weeks (Oshima et al., 2007).

The development of the inner ear from the otic placode is a highly regulated process in which a patch of ectoderm is specified to become a specialized region that invaginates to form the otocyst and, through a complex set of steps, gives rise to the auditory and vestibular organs of the inner ear (Fekete and $\mathrm{Wu}, 2002$; Barald and Kelley, 2004; Ohyama et al., 2006). Progenitor cells in

Received Dec. 7, 2010; revised March 8, 2011; accepted March 23, 2011.

Author contributions: S.-J.J., M.F., A.S.B.E., and S.-C.K. designed research; S.-J.J., M.F., and S.-C.K. performed research; S.-J.J., M.F., A.S.B.E., and S.-C.K. analyzed data; A.S.B.E. wrote the paper.

This work was supported by grants from the National Institute on Deafness and Other Communicative Disorders (R01 DC007174 and P30 DC05209) and from the Harvard Neurodiscovery Center. We thank Oksana Berezovska, John Kessler, Haeyoung Suh-Kim, and Federico Kalinec for cells and plasmids.

*S.J.J. and M.F. contributed equally to this work.

Correspondence should be addressed to AlbertS. B. Edge, Eaton-Peabody Laboratory, Massachusetts Eye and Ear Infirmary, 243 Charles Street, Boston, MA 02114. E-mail: albert_edge@meei.harvard.edu.

M. Fujioka's present address: Department of Otolaryngology, Keio University School of Medicine, Tokyo, 1608582 , Japan.

DOI:10.1523/JNEUROSCI.6366-10.2011

Copyright $\odot 2011$ the authors $\quad 0270-6474 / 11 / 318351-08 \$ 15.00 / 0$ the otocyst differentiate into the sensory cells and neurons that will provide innervation for the sensory organs (Rubel and Fritzsch, 2002). Notch signaling plays a key role in designating the prosensory regions in the otocyst (Lanford et al., 1999; Daudet and Lewis, 2005) and in the differentiation of neuronal and epithelial precursors to neurons (Adam et al., 1998; Lewis et al., 1998), hair cells, and supporting cells (Lanford et al., 1999; Zine et al., 2001; Daudet and Lewis, 2005; Brooker et al., 2006).

Notch helps to determine cell fate by regulating downstream factors including bHLH transcription factors (Lo et al., 2002). Notch signaling is important for the differentiation of specific progeny from stem cells (Grandbarbe et al., 2003; Akagi et al., 2004; Jadhav et al., 2006; Yaron et al., 2006; Wheeler et al., 2008). Inner ear stem cells followed a cell-intrinsic program during differentiation of inner ear cell types (Martinez-Monedero et al., 2008), similar to neural stem cells from the CNS that recapitulated timed differentiation of cortical cells (Shen et al., 2006). Perturbing the Notch signal should alter the ratio of cell types differentiating from inner ear stem cells if it is part of an inherent differentiation program. Notch signaling regulates expression of the neural stem cell marker, Sox2 (Dabdoub et al., 2008), which we have found to be expressed in the inner ear stem cells (Martinez-Monedero et al., 2008) and is a marker for both neural and sensory progenitors in the developing inner ear (Dabdoub et al., 2008; Puligilla et al., 2010).

We asked whether inner ear stem cells were influenced by Notch signaling either in their maintenance and proliferation or in the steps leading to their differentiation to a variety of inner ear cell types. We show that inner ear stem cells can be induced to 
differentiate into hair cells by blockade of Notch signaling by a pathway that is dependent on expression of bHLH transcription factor, Math1 (mouse Atoh1). Once the stem cells have entered a sensory path, Notch retains the progenitors as supporting cells, preventing their differentiation to hair cells. A neuronal fate appears to be selected for cells that show continued expression of Notch. Thus, in addition to increasing proliferation, Notch signaling is responsible for key fate decisions by these cells.

\section{Materials and Methods}

Isolation of inner ear spheres. The utricles of 1to 3-d-postnatal C57BL/6 or Math1-nGFP mice of both sexes (Lumpkin et al., 2003) were dissected and collected. After careful removal of the nerve trunk and mesenchymal tissues, the utricles were trypsinized and dissociated. Dissociated cells were centrifuged, and the pellet was resuspended and filtered through a 70 $\mu \mathrm{m}$ cell strainer (BD Biosciences DiscoveryLabware) in DMEM/F12 medium with N2/B27 supplement, EGF (20 ng/ml), IGF1 (50 ng/ml), bFGF (10 ng/ml), and heparan sulfate (50 ng/ $\mathrm{ml}$ ) (Sigma). The single cells were cultured in nonadherent Petri dishes (Greiner Bio-One) to initiate clonal growth of spheres ( $\mathrm{Li}$ et al., 2003a; Martinez-Monedero et al., 2008).

Spheres that formed after 2-3 d in culture were passaged every $4-6 \mathrm{~d}$. The spheres were centrifuged, and the pellet was mechanically dissociated with pipette tips and resuspended in medium. Passage 3-4 spheres were used for experiments described here. These cells are negative for hair cell markers (Oshima et al., 2007) before the initiation of differentiation.

For differentiation, floating spheres were transferred to fibronectincoated 4 well plates (Greiner Bio-One) as described before (Oshima et al., 2007). Attached spheres were differentiated for 5-7 d in DMEM/F12 medium with N2/B27 supplement but without growth factors. Identification of differentiating cells from the inner ear stem cells has been done previously based on expression of markers for neurons: $\beta$-III tubulin, GluR2, TrkC (Martinez-Monedero et al., 2008); hair cells: myosin VIIa, parvalbumin 3, espin, F-actin (Li et al., 2003b; Oshima et al., 2007; Martinez-Monedero et al., 2008); and supporting cells: Musashi and p27Kip1 (Oshima et al., 2007; Martinez-Monedero et al., 2008). A $\gamma$-secretase inhibitor (L-685458, Sigma) was added at $1 \mu \mathrm{g} / \mathrm{ml}$ on the day following cell attachment. In experiments to assess proliferation, $10 \mu \mathrm{M}$ BrdU was added to the medium $2 \mathrm{~d}$ after the initiation of differentiation. The next day, the BrdU-containing medium was replaced with new medium and cells were cultured for $3 \mathrm{~d}$.

Flow cytometry after transfection. Floating spheres were transferred to fibronectin-coated 4 well culture dishes for attachment, and the next day, attached spheres were transfected with Notch intracellular domain (NICD)-GFP (a generous gift from Oksana Berezovska, Massachusetts General Hospital, Boston, MA) containing the NICD coding sequence linked to GFP under an EF1 $\alpha$-promotor in the pTracer-EF vector (Invitrogen) or with a control vector containing GFP using Lipofectamine 2000 (Sigma) in Opti-MEM medium (Invitrogen). The cells were exposed for $6 \mathrm{~h}$ to $2 \mu \mathrm{g} / \mathrm{ml}$ NICD-GFP. The transfected stem cells were further differentiated for 5-6 d in DMEM/F12 medium with N2/B27 supplement without growth factors.

For FACS analysis, spheres were detached by trypsinization $2 \mathrm{~d}$ after transfection with NICD-GFP and triturated. This procedure completely dissociated the cells (as confirmed by microscopic examination). NICD-
$\mathrm{GFP}^{+}$cells were sorted on a FACSAria cell sorter (BD Biosciences). From

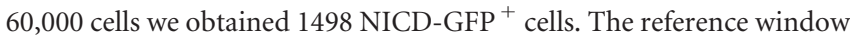
was set so that fluorescence from the NICD-GFP ${ }^{-}$cells was $<2 \%$.

RNAi silencing. The utricle-derived stem cells were transfected with siRNA (Dharmacon) as for the other transfections, but using Gene Silencer (Genlantis) according to the manufacturer's instructions. After transfection, the cells were washed twice and then cultured in DMEM/ F12 (1:1) with N2/B27 for 4-5 d. The final working concentration of RNAi was $200 \mathrm{~nm}$ for $6 \mathrm{~h}$. The transfected cells were easily identified by cotransfected fluorescent siRNA. Transfection efficiency was $82 \%$. The same concentration of nontargeting RNAi (Dharmacon, ON-TARGETplus nontargeting siRNA, size-matched and selected for lack of an effect on gene expression) was used in parallel as a control, and gene silencing was assessed by quantitative $(\mathrm{q})$ reverse transcriptase (RT)-PCR.

$q R T-P C R$. Total RNA was extracted from siRNA-treated cells and cells sorted by flow cytometry with the RNeasy Mini Kit (Qiagen) according to the manufacturer's instructions. Equal numbers of NICD-GFP ${ }^{-}$cells were used as a control for the $\mathrm{GFP}^{+}$cells obtained by cell sorting, and siRNA controls were cells treated with nontargeting siRNA in parallel with the siRNA for the target. For reverse transcription, SuperScript II (Invitrogen) was used with random hexamers. The reverse transcription conditions were $25^{\circ} \mathrm{C}$ for $10 \mathrm{~min}$ followed by $37^{\circ} \mathrm{C}$ for $60 \mathrm{~min}$. The reaction was terminated at $95^{\circ} \mathrm{C}$ for $5 \mathrm{~min}$. The cDNAs were mixed with Taqman Gene Expression Mastermix (Applied Biosystems) and Math1, Ngn1, and NeuroD primers according to the manufacturer's instructions. Samples were analyzed in 96 wells in triplicate by qPCR (Applied Biosystems 7900HT), and PCR thermal cycling conditions were as follows: initial denaturation at $95^{\circ} \mathrm{C}$ for $2 \mathrm{~min}$, denaturation at $95^{\circ} \mathrm{C}$ for $15 \mathrm{~s}$, annealing/extension at $60^{\circ} \mathrm{C}$ for $1 \mathrm{~min}$ for 45 cycles. Conditions were kept constant for each primer. Gene expression was calculated relative to $18 \mathrm{~S}$ RNA, and the amount of cDNA applied was adjusted so that the $C_{\mathrm{t}}$ value for 18S RNA was between 10 and 13 . 

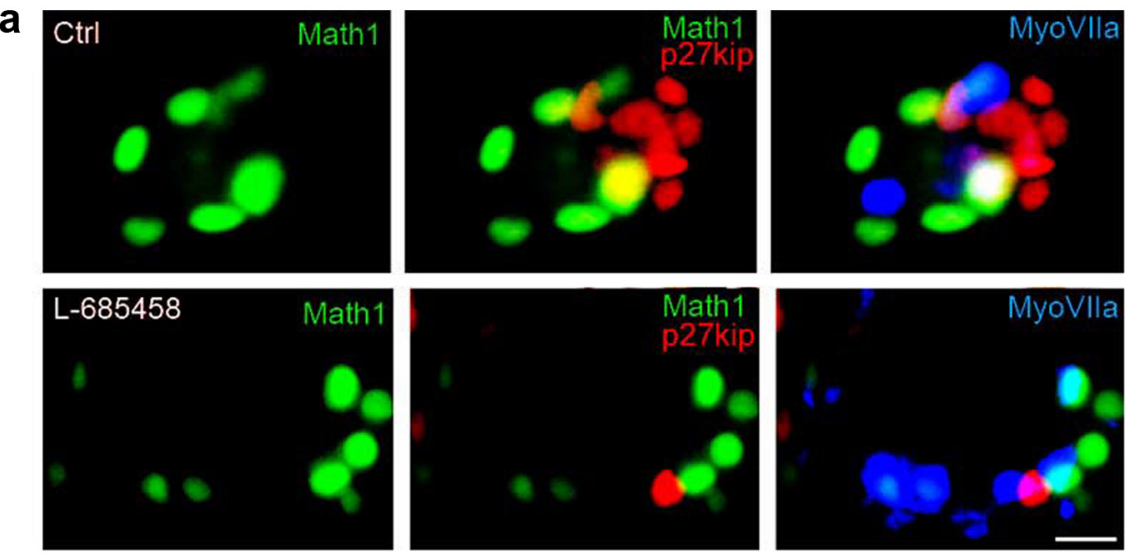

b

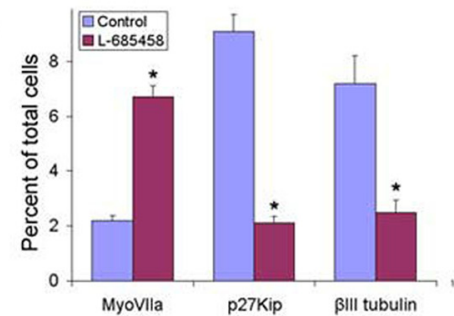

C

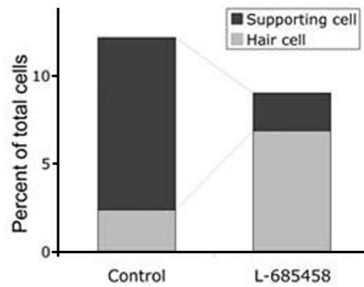

d

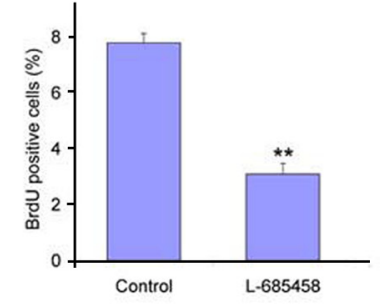

Figure 2. Hair cells were formed at the expense of supporting cells in sensory patches in inner ear spheres. $\boldsymbol{a}$, Treatment of the inner ear spheres with a $\gamma$-secretase inhibitor for $5 \mathrm{~d}$ increased the percentage of Math1-nGFP (green)- and myosin VIlla (blue)positive cells and decreased the percentage of p27Kip1 (red)-positive cells. Scale bar, $10 \mu \mathrm{m}$. $\boldsymbol{b}$, The percentage of hair cells (MyoVIla) that were differentiated from the inner ear spheres was increased and the percentage of supporting cells (p27Kip) and neurons ( $\beta$ III tubulin) was decreased by treatment with the $\gamma$-secretase inhibitor. $\boldsymbol{c}$, The data from $(\boldsymbol{b})$ yielded an increased percentage of hair cells and decreased percentage of supporting cells but the sum of hair cells and supporting cells was only slightly decreased after treatment with the $\gamma$-secretase inhibitor, L-685458. $\boldsymbol{d}$, Inhibition of Notch with L-685458 decreased the percentage of proliferating cells measured by labeling with BrdU. The total cell number was acquired by the counting of DAPI-positive cells (4681 cells from the control and 5882 cells treated with $\gamma$-secretase inhibitor). Error bar is SE. ${ }^{*} p<0.05,{ }^{* *} p<0.01$.

Luciferase reporter assays. Luciferase reporter assays were performed with HEK293T cells, Neuro2a cells, and HEI-OC1 cells (a kind gift from Federico Kalinec, House Ear Institute, Los Angeles, CA). Cells were transfected using FuGENE6 (Roche) following the manufacturer's protocol. Twenty-four hours after transfection, cell lysates were collected and luciferase assays were performed using Dual-Luciferase Reporter Assay System (Promega). The following vectors were used: Math1luciferase, consisting of a $1.4 \mathrm{~kb}$ J2X enhancer of Atoh 1 that is sufficient for endogenous Atoh1 expression during development (Helms et al., 2000), Ngn1-luciferase [a kind gift from John Kessler (Northwestern University, Chicago, IL) in ptkRLuc (Promega)], NICD-GFP, pcDNA3.1/His(B)-Ngn1 (a generous gift from Haeyoung Suh-Kim, Ajou University, Suwon, South Korea), and pTRACK-CMV-Math1. All data were normalized to the Renilla luciferase signal.

Immunohistochemistry. Cells were fixed for $10 \mathrm{~min}$ with $4 \%$ paraformaldehyde in PBS. Immunostaining was initiated by rehydrating and blocking the sections for $1 \mathrm{~h}$ with $0.1 \%$ Triton X-100 in PBS supplemented with $1 \%$ BSA and $5 \%$ goat serum (PBT1). Fixed and permeabilized cells were incubated overnight in PBT1 with polyclonal antibody to myosin VIIa (Affinity Bioreagents), monoclonal antibody to TuJ (Sigma), polyclonal antibody to p27Kip1 (Neomarkers), monoclonal antibody to annexin (Sigma), or monoclonal antibody to BrdU (Accurate
Chemicals). Samples were washed three times for 20 min with PBS. Primary antibodies were detected with secondary antibodies conjugated with FITC, TRITC, or Cy-5 (Jackson ImmunoResearch). Secondary antibody alone was used for a negative control. The samples were counterstained with DAPI (Vector Laboratories) or TOTO3 (Invitrogen) for $10 \mathrm{~min}$ and viewed by epifluorescence microscopy (Axioskop 2 Mot Axiocam, Zeiss) or confocal microscopy (TCS, Leica).

Quantification and statistical analysis. Cell counting was performed with MetaMorph software. The cell number was determined from DAPI- or TOTO3-positive nuclei. Repeat cell counting gave a test variation of $<1 \%$. Data are reported as mean $\pm \mathrm{SE}$, and Student's $t$ test was used for statistical analysis.

Western blot. Cells were transfected with the NICD-GFP plasmid using FuGENE6 (Roche), following the manufacturer's protocol, and harvested after $48 \mathrm{~h}$. After incubation for 15 min in RIPA buffer (Invitrogen) on ice, samples were centrifuged and the supernatant was collected. Twenty micrograms of protein was subjected to SDS-PAGE on NuPage 4-12\% gradient polyacrylamide gels (Invitrogen) and transferred to a nitrocellulose membrane for immunoblotting. Primary antibodies were rabbit anti-Ngn1 (1:300; Millipore Bioscience Research Reagents) and mouse anti- $\beta$-actin monoclonal antibody (1:1000; Sigma); secondary antibodies were HRP-conjugated goat antirabbit and mouse (both at 1:10,000; Jackson ImmunoResearch Laboratories). Blots were developed with the ECL detection kit (GE Healthcare Bio-Sciences). Proteins in the blots were quantified using the Quantity One program (Bio-Rad).

\section{Results}

Inhibition of $\boldsymbol{\gamma}$-secretase resulted in increased differentiation of hair cells

\section{from inner ear stem cells}

Under differentiating conditions in culture for 5-10 d, inner ear stem cells are converted from hair cell marker-negative into a variety of cell types that include sensory hair cells (Martinez-Monedero et al., 2008). The hair cells have been shown in previous studies to have functional ion channels of embryonic day 15 utricular hair cells (Oshima et al., 2007), and they express multiple markers of hair cells such as myosin VIIa, Math1, and parvalbumin 3 (Oshima et al., 2007; MartinezMonedero et al., 2008). Neurons have also been shown to differentiate from the spheres and can be labeled for markers such as $\beta$-III tubulin, peripherin, calretinin, and glutamate receptors, as well as becoming physiologically active and responsive to glutamate (Martinez-Monedero et al., 2008). Notch signaling exerts a powerful effect on maintenance of stem cell compartments, and we wanted to determine its role in proliferation or differentiation of inner ear stem cells. Differentiation of inner ear stem cells in the presence of a $\gamma$-secretase inhibitor increased the percentage of hair cells compared with a control, as detected by immunostaining for myosin VIIa and Math1 (Fig. 1). The percentage of hair cells in the presence of the inhibitor was $6.68 \pm 0.49 \%$ compared with $2.23 \pm 0.36 \%$ in its absence. 
Hair cells in inner ear spheres were formed at the expense of supporting cells

Hair cells in spheres appear to form as a part of a sensory patch (Fekete and Wu, 2002; Barald and Kelley, 2004; Oshima et al., 2007), and had adjacent supporting cells, providing a cell-cell relationship similar to that in vivo. Supporting cells have been identified in previous studies of cochlear spheres by their expression of markers, such as p27Kip1 and Musashil (Oshima et al., 2007), and have also been shown to arise adjacent to newly generated hair cells in embryonic sensory epithelium by jagged 1 expression (Woods et al., 2004). We followed both hair cells and supporting cells in differentiating spheres to see whether Notch signaling influenced the ratio of the two cell types as it does in vivo. Treatment with the $\gamma$-secretase inhibitor at the time of differentiation resulted in an increase in hair cell formation that was correlated with a decrease in the number of supporting cells in the sensory patch (Fig. $2 a, b$ ), as measured by expression of p27Kip1 (Fig. 2a). Additional labeling for Sox 2 and jagged1 overlapped extensively with $\mathrm{p} 27 \mathrm{Kip} 1$, confirming the identity of the supporting cells (data not shown). The decrease was significant, but the sum of hair cells and supporting cells was only slightly decreased (Fig. 2c) as a result of a decrease in proliferation during the $5 \mathrm{~d}$ of differentiation (Fig. $2 d$ ).

Treatment with the $\gamma$-secretase inhibitor blocked differentiation of $\beta$-III tubulin-positive cells (Fig. $2 b$ ). This suggested that neuronal differentiation that led to $\beta$-III tubulin-positive cells from the spheres required Notch signaling.

Inhibition of Notch signaling increased differentiation of hair cells by an effect on Math1

To assess the role of bHLH transcription factors in the cell fate decisions that appeared to be mediated by Notch, we assessed the expression of Math 1 after treatment with the $\gamma$-secretase inhibitor. The inner ear progenitor cells treated with a $\gamma$-secretase inhibitor upregulated expression of Math1 (Fig. 3a). To examine the mechanism of increased hair cell formation in inner ear neurospheres after $\gamma$-secretase inhibition, expression of Math1 was silenced by transfection of spheres with siRNA to Math1. The increase in Math 1 could be blocked by siRNA to Math 1 by $80.5 \%$ (relative expression of 1.46 compared with 7.46) based on qRTPCR. Silencing Math1 in neurospheres treated with L-685458 (Fig. $3 b$ ) inhibited conversion of inner ear neurospheres to myosin VIIa-positive cells by $75.9 \%$ (Fig. $3 c$ ). Although the activity of $\gamma$-secretase inhibitors is not limited to Notch, the increase in hair cell percentage and its dependence on Math1 expression is very likely an effect of the inhibitor on Notch.

\section{Notch increased expansion and differentiation of neuronal progenitors}

Inhibition of Notch signaling increased hair cell differentiation but resulted in a decreased number of cells expressing $\beta$-III tubulin (Fig. 2b). To investigate the effect of Notch signaling on the inner ear stem cells, we overexpressed NICD fused to GFP by transient transfection. Transfection with NICD increased overall proliferation $(35.2 \pm 0.9 \%$ of NICD-transfected cells were BrdU positive compared with $7.10 \pm 0.63 \%$ of total cells; Fig. $4 a$ ), in accordance with the decreased proliferation after treatment with a $\gamma$-secretase inhibitor (Fig. $2 d$ ). This indicated that the Notch pathway played a role in increasing cell division of the inner ear stem cells. Analysis after $5 \mathrm{~d}$ in culture showed that $22.1 \pm 3.1 \%$ of NICD-positive cells expressed $\beta$-III tubulin, a marker previously associated with the neuronal lineage in inner ear sphere-derived neurons (Martinez-Monedero et al.,
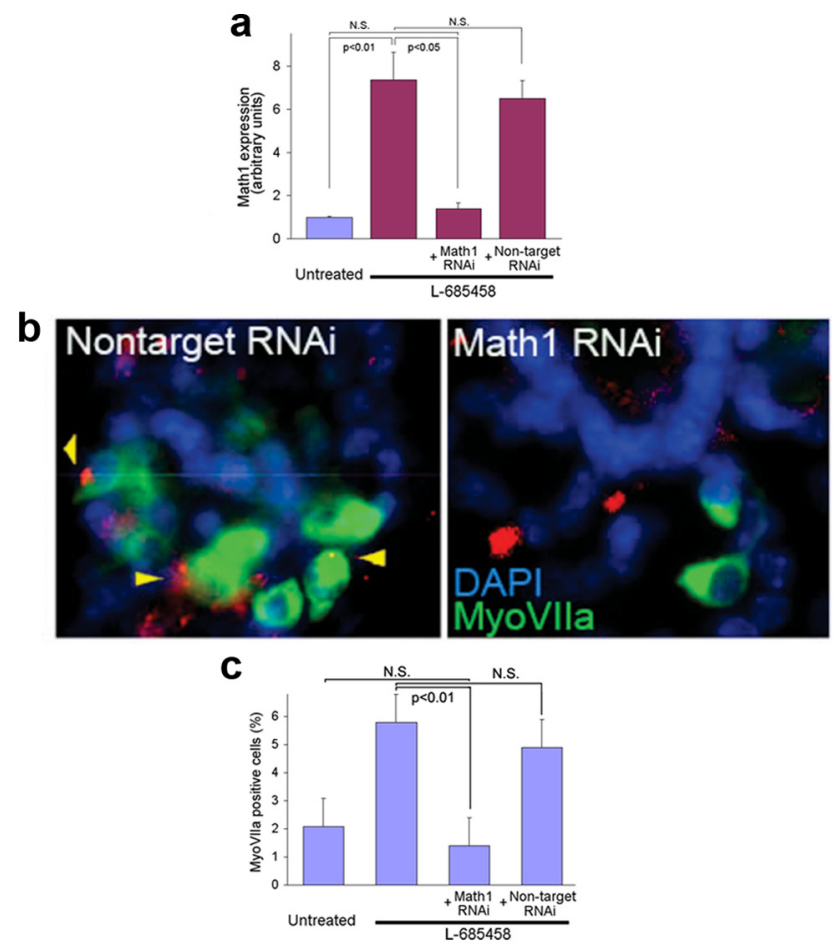

Figure 3. Inhibition of $\gamma$-secretase increased hair cell differentiation by upregulating Math1. $\boldsymbol{a}$, Expression of Math1 in inner ear stem cells was increased after treatment with a $\gamma$-secretase inhibitor and could be blocked by siRNA to Math1 but not by a nontargeting siRNA based on qRT-PCR. $\boldsymbol{b}$, Differentiation of hair cells (myosin Vlla, green) induced by the $\gamma$-secretase inhibitor was unaffected by nontargeting siRNA but was inhibited by siRNA directed at Math1. Labeled siRNA (red, arrowheads) was added to follow transfection. Blue, DAPI. Scale bar, $15 \mu \mathrm{m}$. c, The percentage of hair cells obtained after treatment with the $\gamma$-secretase inhibitor was significantly decreased by Math1 RNAi. The percentage was reduced to the level of the untreated control but was not decreased by nontargeting RNAi. Error bars, SE; * $p<0.05$, ** $p<0.01$.

2008), and had a neuronal morphology (Fig. 4b). None of the GFP-positive cells stained for myosin VIIa (Fig. 4c), indicating that differentiation to hair cells was strongly inhibited in sphere cells transfected with NICD. It therefore seemed that continued Notch signaling blocked hair cell differentiation but did not prevent progenitor cells from entering a neuronal lineage.

To understand how signaling by Notch influenced the choice of inner ear stem cell fate, we sorted the NICD-GFPpositive inner ear stem cells by flow cytometry and assessed expression of several neural progenitor genes by qRT-PCR (Fig. 4d,e). Sharply increased expression of Hes5 (Fig. 4e) confirmed that the cells were successfully transfected with NICD and that the NICD was active. An increase in a marker of both sensory and neuronal lineages, Sox2, indicated that this gene was downstream of Notch signaling in the inner ear progenitors. A concomitant increase was found in proneural transcription factor, Ngn1, suggesting a possible explanation for the increased expression of $\beta$-III tubulin in these cultures, since Ngn 1 is a marker of neuronal progenitors in the inner ear and can also determine the fate of CNS neurons. There was no significant change in Math1 expression (Fig. 4e) in the cells obtained by sorting.

To test whether NICD could directly increase Ngn1 expression, we used a cochlear cell line, HEI-OC1. A luciferase vector with a $1.5 \mathrm{~kb}$ regulatory region $5^{\prime}$ of the coding sequence for Ngn 1 (Kan et al., 2004) was transfected into these cells. This enhancer 
a

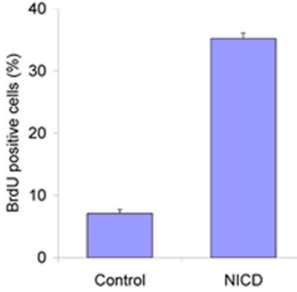

b
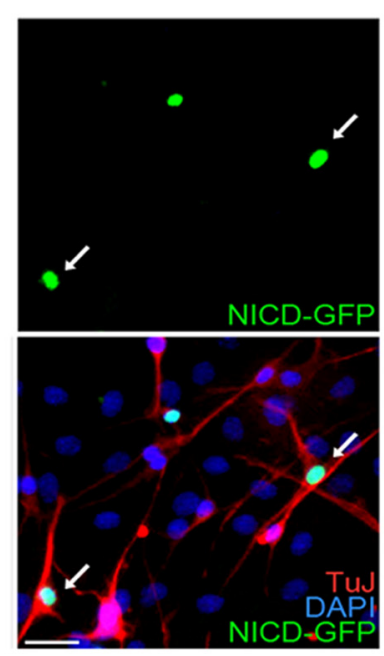

C
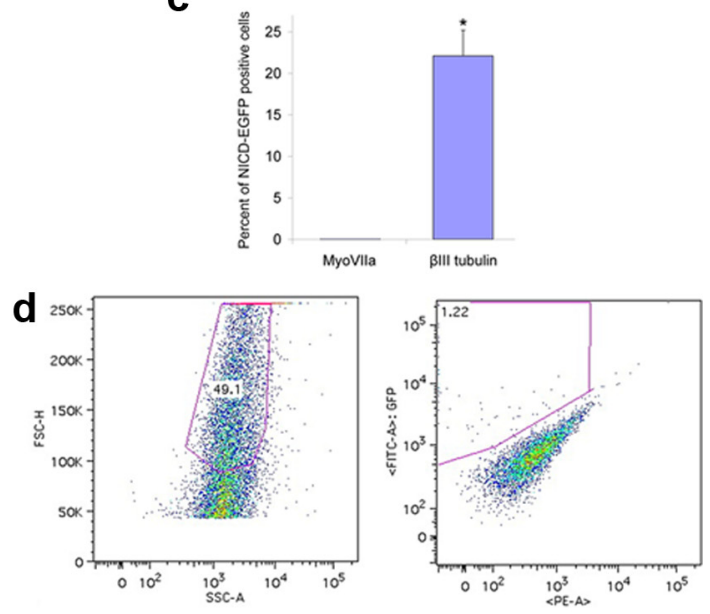

e

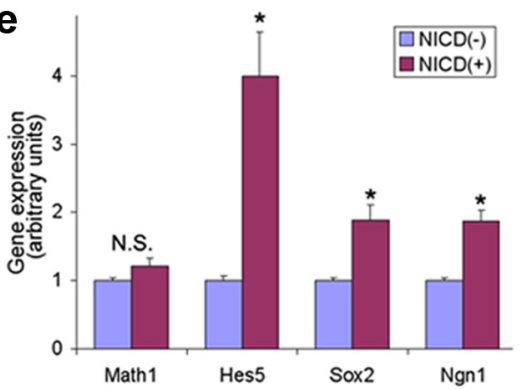

Figure 4. Transfection of inner ear stem cells with the intracellular domain of Notch increased cell division and affected expression of $\mathrm{Ngn} 1$ and Sox2. $\boldsymbol{a}$, Overexpression of NICD increased the percentage of dividing cells. $\boldsymbol{b}$, Staining of the differentiated cells with an antibody (TuJ) to $\beta$-III tubulin showed that some labeled cells (red) had nuclear labeling with NICD-GFP (arrowheads; blue, DAPI). Scale bar, $15 \mu \mathrm{m}$. c, The percentage of cells that labeled for both NICD and $\beta$-III tubulin is shown. None of the cells that labeled for NICD were colabeled for myosin VIla. $\boldsymbol{d}$, The stem cells were transfected with NICD-GFP and were selected as shown (forward vs side-scatter). Those cells with strong green fluorescence were purified by flow cytometry. $\boldsymbol{e}$, The sorted NICD-GFPpositive cells were subjected to qRT-PCR and had increased expression of Hes5, Sox2 and Ngn1 genes. Error bars, SE. ${ }^{*} p<0.05$, significant difference.

was shown to be sufficient for expression of Ngn1 in the relevant tissues (Murray et al., 2000). Cotransfection of NICD increased expression of the reporter, whereas expression from the Math1 enhancer was not affected (Fig. 5a). Examination of the 5' Ngn1 enhancer indicated the occurrence of an RBP-J binding site $0.6 \mathrm{~kb}$ upstream of the coding sequences (Fig. $5 b$ ). The site was an exact match to the canonical RBP-J site (Fig. $5 b$; the RBP-J binding sequence is labeled a TP-1 site). This raised the possibility that overexpression of Notch raised expression of Ngn1 through binding of NICD to this site. To test whether the site was functional, we transfected NICD into cells containing a mutated RBPj binding site. The luciferase signal from the enhancer with mutated RBPj site (Fig. $5 c$ ) was decreased significantly but not abolished, suggesting that direct binding of NICD did not account for the entire activation of the Ngn1 enhancer. We then assessed the effect of Sox2, a gene that was activated along with Ngn1 (Fig. 4f) by NICD overexpression, by experiments in which we silenced Sox 2 with RNAi. The increase in Ngn1 expression seen after transfection of NICD was almost completely abolished by the siRNA that prevented expression of Sox2 in combination with the mutation of the RBPj binding site (Fig. $5 c$ ). To assess whether increased expression of Sox 2 affected Ngn1 expression as expected from the silencing experiment, we overexpressed Sox2 in HEI-OC1 cells. The Ngn1-luciferase vector showed an increase in activation (Fig. 5d). Thus, indirect activation of Ngn 1 by Sox 2 in combination with direct binding of NICD to the Ngn1 enhancer accounted for activation of Ngn1. Increased expression of $\mathrm{Ngn} 1$ in response to NICD in HEI-OC1 cells was also confirmed at the level of the protein by Western blotting (Fig. $5 f$ ).

\section{Effect of Ngn1 on neuronal differentiation from inner ear neurospheres}

Silencing of Ngn1 inhibited the conversion of inner ear neurospheres to $\beta$-III tubulinpositive cells (Fig. $6 a$ ). The percentage of inner ear sphere cells that stained for $\beta$-III tubulin was reduced from $6.2 \%$ to $2.2 \%$ by RNAi to Ngn1. Silencing of Math1 had a smaller effect that did not achieve significance (Fig. 6b).

To determine whether the NICDinduced neuronal differentiation was mediated by Ngn1, we treated inner ear stem cells with the siRNA at the same time as the transfection of the cells with NICD (Fig. 6c). The percentage of cells that differentiated to $\beta$-III tubulin-positive cells was sharply decreased by siRNA directed at Ngn1. The extent of inhibition was $70.2 \%$ with siRNA to Ngn1 (Fig. 6d). The effect of siRNA to Math1 was not significant.

\section{Discussion}

We have shown in these studies that inner ear stem cell proliferation and differentiation are regulated by the Notch pathway. Math1 and Ngn1 had opposing effects on cell fate, leading, respectively, to sensory (hair cell and supporting cell) or neuronal pathways of differentiation, and, by its effect on proliferation of the stem cells, as well as on the expression of these transcription factors, Notch signaling played a critical role in the cell fate decisions of inner ear stem cells.

Inhibition of Notch signaling increased the percentage of progenitor cells that differentiated as hair cells. To determine the cause of the increased hair cell differentiation, we assessed the influence of Notch pathway inhibition on Math1. Math1 expression was increased by inhibition of Notch signaling in the inner ear progenitors, and blocking expression of this bHLH transcription factor prevented the $\gamma$-secretase-stimulated differentiation, indicating that Math1 was necessary and sufficient to differentiate the progenitors to hair cells. Thus, by preventing expression of Math1, Notch signaling appeared to preserve the progenitor cells in an undifferentiated state. A key role of Math1 in determining whether these cells differentiate into hair cells is consistent with its known role in vivo and with the observation that Math $1^{-1-}$ mice lack hair cells (Bermingham et al., 1999). The differentiation of hair cells took place through the intermediate formation of cells positive for p27Kip1 and negative for Math1 and myosin VIIa that appeared to be supporting cells. The alternating arrangement of hair cells and supporting cells in vivo depends on the downregulation of Notch in hair cells and is a classic example of lateral inhibition (Lanford et al., 1999; Eddison et al., 2000; Kiernan et al., 2005a; Brooker et al., 2006). The conversion of supporting cells to hair cells was strikingly similar to the situation 

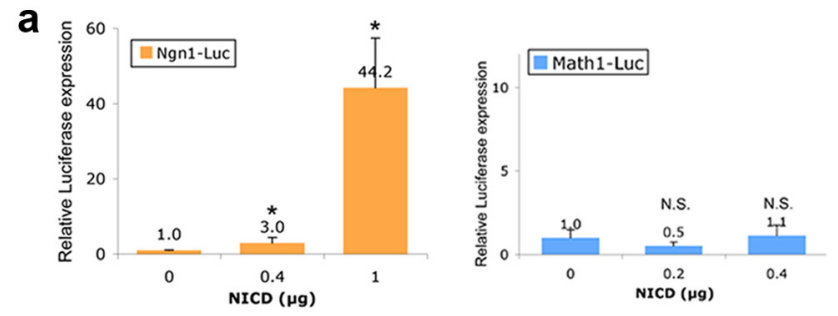

b

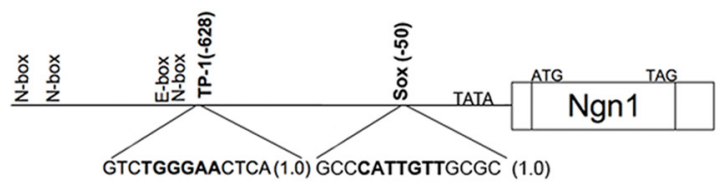

C

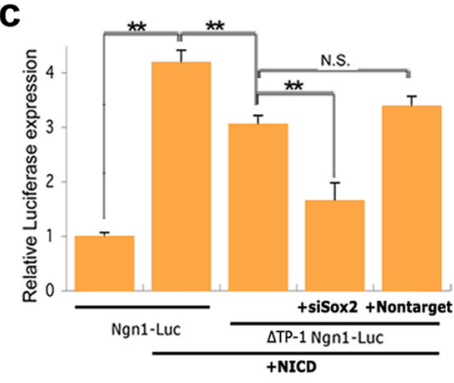

d

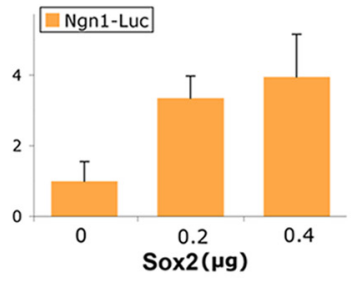

e
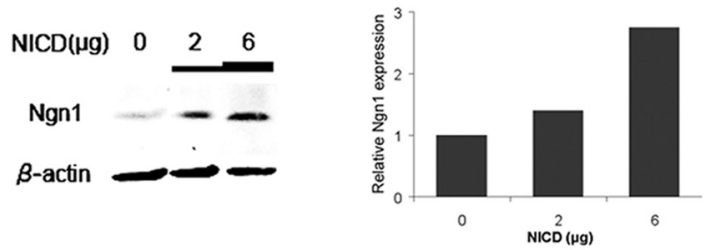

Figure 5. NICD increased Ngn1 expression by direct and indirect mechanisms. $\boldsymbol{a}$, NICD had no effect on Math1 expression but increased expression of luciferase from the Ngn 1 construct in HEI-OC1 cells. $\boldsymbol{b}$, Map of the $1.5 \mathrm{~kb}$ regulatory region of the $\mathrm{Ngn} 1$ gene. Sequences are followed by matrix match scores for the indicated binding sites. $c$, Mutation of the TP-1 site decreased the activation of the Ngn1 enhancer by NICD. $\boldsymbol{d}$, The Ngn1 enhancer was activated by overexpression of Sox2 based on luciferase assay. $\boldsymbol{e}$, Western blot showed increased expression of Ngn1 after overexpression of NICD in HEI-OC1 cells. Error bar, $\mathrm{SE} .{ }^{*} p<0.05$, significant difference.

in embryonic organ of Corti, in which supporting cells can be converted to hair cells by treatment with a $\gamma$-secretase inhibitor (Yamamoto et al., 2006). Supporting cells are precursors for hair cells in lower vertebrates, and this conversion can be increased by inhibition of Notch signaling (Stone and Cotanche, 2007; Daudet et al., 2009).

By maintaining the stem cells in an undifferentiated state, NICD favored the selection of a neuronal lineage. An increase in cells in the neuronal lineage relative to sensory cells was apparent from the subsequent differentiation. Expansion of neural progenitors in the developing inner ear may also be controlled by Notch (Lewis et al., 1998), and differentiation of progenitors to neurons is regulated by Ngn1 (Rubel and Fritzsch, 2002; Fritzsch et al., 2005; Matei et al., 2005; Raft et al., 2007) in opposition to sensory cell formation, which is controlled by Math1. Increased neuronal fate of inner ear stem cells could be attributed in part to Ngn1 expression in the cochlear spheres, which was found after NICD transfection and sorting. Increased activation of the Ngn1 enhancer and upregulation of Ngn1 protein was confirmed by analysis of Ngn1 after NICD overexpression in cochlear cells.

A binding site for RBP-J in the Ngn1 promoter/enhancer indicated that there might be a direct route for the activation of Ngn1 by Notch. The same regulatory region contained a binding site for Sox 2, a transcription factor that is regulated by Notch, and
Sox 2 expression positively regulated Ngn1, whereas silencing of Sox 2 negatively regulated Ngn1. Mutation of the RBP-J binding site did indeed inhibit the activation of luciferase from a reporter gene containing the Ngn1 enhancer. However, the effect was small and indicated that Sox 2 was more important in the regulation of Ngn1 than NICD itself. The role of Sox 2 in this increase was surprising. Sox 2 is thought to maintain the pluripotency of neural progenitors in the CNS through inhibition of bHLH transcription factors (Bylund et al., 2003; Graham et al., 2003). Neural differentiation can, however, be induced by upregulation of proneural transcription factors by Sox2 (Van Raay et al., 2005), and that upregulation can include Ngn1 in the case of Sox1 (Kan et al., 2004). Sox2 expression after neural differentiation is limited by negative feedback regulation by proneural factors once their expression is activated (Bylund et al., 2003; Graham et al., 2003), and a mechanism in which each step in the pathway inhibits the preceding step and activates the subsequent step may insure the progress of neural differentiation (Agathocleous et al., 2009). In addition to the NICD binding site, the Ngn1 enhancer contains N-boxes for binding of Hes1 and Hes5, which act downstream of Notch to inhibit bHLH transcription factors, and E-boxes for binding of other bHLH transcription factors, creating an opportunity for complex regulation in a particular developmental context. RBP-J can also positively regulate transcription independently of Notch by binding to the RBP-J site in a complex with Tbf1 (Henke et al., 2009). Timing- and tissue-specific regulation of bHLH factors is well documented and is illustrated by this system in which Notch signaling strength seems to function in sensory and neuronal progenitor fate choice. Our finding may also explain the observation that Ngn1 was less sensitive than Mash1 to Notch downregulation in the neural crest (Lo et al., 2002).

Notch signaling, in general, maintains progenitors in the nervous system in a nondifferentiated state but participates in specification of glial fate (Grandbarbe et al., 2003; Jadhav et al., 2006; Kageyama et al., 2008; Imayoshi et al., 2010). The pattern of cell type specification from inner ear stem cells is similar to that in the developing sensory organ of Drosophila (Eddison et al., 2000; Cau and Blader, 2009). In the neural versus epidermal fate decision in sensory organ precursors, Notch selects an epidermal fate; in subsequent neural cell type specification, Notch helps to select a neural subtype. Notch increased proliferation of neuronal precursors, thus increasing their number, and may play a permissive role that allows neuronal differentiation from Notch-activated cells later in their life cycle; a role for Notch in cochlear stem cell proliferation was also demonstrated previously (Savary et al., 2008). Notch signaling is required at both early and late stages of neural progenitor specification and differentiation in the Drosophila eye (Zhang et al., 2005; Wheeler et al., 2008) and in the embryonic development of retinal ganglion cells and horizontal cells (Yaron et al., 2006). The initial specification of the prosensory domain in the inner ear requires both Notch and Sox2 (Kiernan et al., 2005b; Dabdoub et al., 2008); however, neural differentiation was favored in sensory epithelial cells after Sox2 overexpression (Puligilla et al., 2010), and hair cell differentiation was decreased.

The stem cells in the inner ear have been shown to be capable of differentiation into most cell types of the mouse cochlea, including hair cells and supporting cells and the neurons that provide their innervation (Li et al., 2003a; Oshima et al., 2007; Martinez-Monedero et al., 2008). Neural and sensory cell progenitors in the otic placode during embryonic development (Fekete and $\mathrm{Wu}, 2002$; Barald and Kelley, 2004; Giraldez and 
a
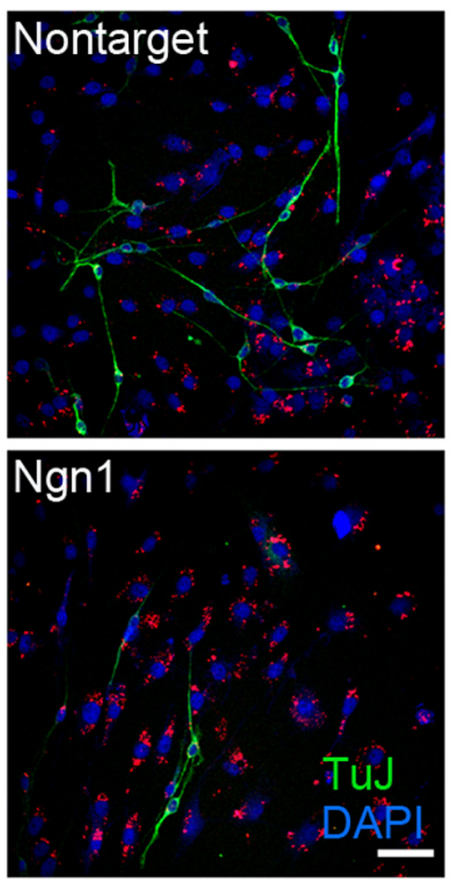

b

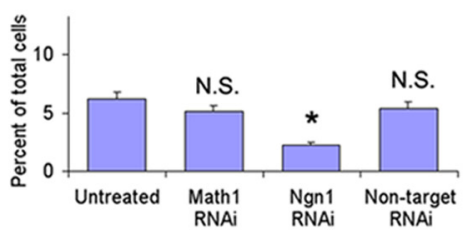

C
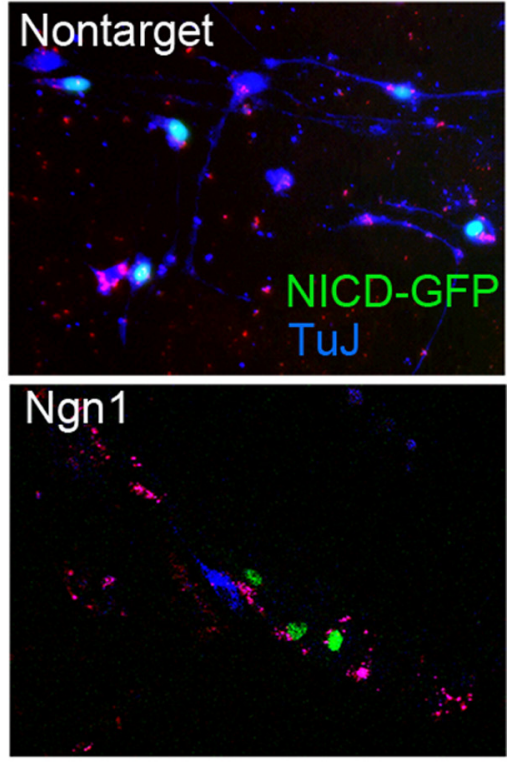

d

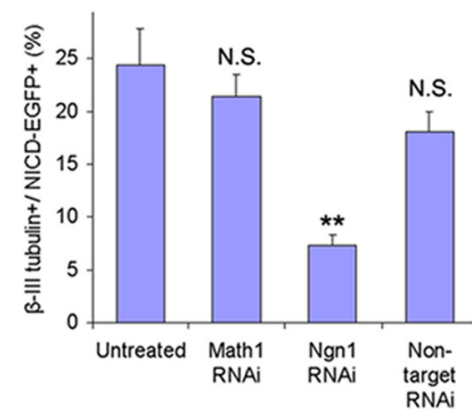

Figure 6. siRNA directed at Ngn 1 inhibited differentiation of inner ear stem cells to $\beta$-III tubulin-positive cells. $\boldsymbol{a}$, Inner ear stem cells treated with siRNA to $\mathrm{Ngn} 1$ compared with nontargeting siRNA (red) had a decreased percentage of TuJ-positive cells (green). Blue, DAPI. Scale bar, $20 \mu \mathrm{m}$. $\boldsymbol{b}$, Ngn1 siRNA inhibited differentiation to $\beta$-III tubulin-positive cells. c, NICD-transfected cells were treated with siRNA, and differentiation to a neuronal morphology was unaffected by a nontargeting sequence ( $\beta$-III tubulinpositive cells shown in blue), but their expression of $\beta$-III tubulin was blocked by siRNA to Ngn1.d, (ells that expressed nuclear GFP were counted for $\beta$-III tubulin after siRNA treatment. Error bar, SE. ${ }^{*} p<0.05$, significant difference.

Fritzsch, 2007) can produce neurons and hair cells after they are separated and cultured apart from the embryo (Adam et al., 1998), and some reports have indicated that single progenitors can give rise to neurons and sensory epithelial cells in the inner ear (Satoh and Fekete, 2005). Progenitors in the otic placode give rise to all cells in the inner ear except the glial cells in the spiral ganglion, which are thought to be derived from neural crest (Neves et al., 2007). Both specification of the sensory domains within the otic placode and later differentiation of the hair cells and supporting cells of the sensory epithelium are dependent on Notch signaling (Lanford et al., 1999; Daudet and Lewis, 2005; Kiernan et al., 2005a). Notch signaling, through its effects on bHLH transcription factors, thus helps to direct the fate of inner ear progenitors to the key cell types of the cochlea.

\section{References}

Adam J, Myat A, Le Roux I, Eddison M, Henrique D, Ish-Horowicz D, Lewis J (1998) Cell fate choices and the expression of Notch, Delta and Serrate homologues in the chick inner ear: parallels with Drosophila sense-organ development. Development 125:4645-4654.

Agathocleous M, Iordanova I, Willardsen MI, Xue XY, Vetter ML, Harris WA, Moore KB (2009) A directional Wnt/beta-catenin-Sox2-proneural pathway regulates the transition from proliferation to differentiation in the Xenopus retina. Development 136:3289-3299.

Akagi T, Inoue T, Miyoshi G, Bessho Y, Takahashi M, Lee JE, Guillemot F,
Kageyama R (2004) Requirement of multiple basic helix-loop-helix genes for retinal neuronal subtype specification. J Biol Chem 279: 28492-28498.

Barald KF, Kelley MW (2004) From placode to polarization: new tunes in inner ear development. Development 131:4119-4130.

Bermingham NA, Hassan BA, Price SD, Vollrath MA, Ben-Arie N, Eatock RA, Bellen HJ, Lysakowski A, Zoghbi HY (1999) Mathl: an essential gene for the generation of inner ear hair cells. Science 284:1837-1841.

Brooker R, Hozumi K, Lewis J (2006) Notch ligands with contrasting functions: Jagged 1 and Delta1 in the mouse inner ear. Development 133:1277-1286.

Bylund M, Andersson E, Novitch BG, Muhr J (2003) Vertebrate neurogenesis is counteracted by Sox1-3 activity. Nat Neurosci 6:1162-1168.

Cau E, Blader P (2009) Notch activity in the nervous system: to switch or not switch? Neural Dev 4:36.

Dabdoub A, Puligilla C, Jones JM, Fritzsch B, Cheah KS, Pevny LH, Kelley MW (2008) Sox2 signaling in prosensory domain specification and subsequent hair cell differentiation in the developing cochlea. Proc Natl Acad Sci U S A 105:18396-18401.

Daudet N, Lewis J (2005) Two contrasting roles for Notch activity in chick inner ear development: specification of prosensory patches and lateral inhibition of hair-cell differentiation. Development 132:541-551.

Daudet N, Gibson R, Shang J, Bernard A, Lewis J, Stone J (2009) Notch regulation of progenitor cell behavior in quiescent and regenerating auditory epithelium of mature birds. Dev Biol 326:86-100.

Eddison M, Le Roux I, Lewis J (2000) Notch signaling in the development of the inner ear: lessons from Drosophila. Proc Natl Acad Sci U S A 97:11692-11699.

Fekete DM, Wu DK (2002) Revisiting cell fate specification in the inner ear. Curr Opin Neurobiol 12:35-42.

Fritzsch B, Matei VA, Nichols DH, Bermingham N, Jones K, Beisel KW, Wang VY (2005) Atoh1 null mice show directed afferent fiber growth to undifferentiated ear sensory epithelia followed by incomplete fiber retention. Dev Dyn 233:570-583.

Giraldez F, Fritzsch B (2007) The molecular biology of ear development“Twenty years are nothing". Int J Dev Biol 51:429-438.

Graham V, Khudyakov J, Ellis P, Pevny L (2003) SOX2 functions to maintain neural progenitor identity. Neuron 39:749-765.

Grandbarbe L, Bouissac J, Rand M, Hrabé de Angelis M, Artavanis-Tsakonas S, Mohier E (2003) Delta-Notch signaling controls the generation of neurons/glia from neural stem cells in a stepwise process. Development 130:1391-1402.

Helms AW, Abney AL, Ben-Arie N, Zoghbi HY, Johnson JE (2000) Autoregulation and multiple enhancers control Math1 expression in the developing nervous system. Development 127:1185-1196.

Henke RM, Savage TK, Meredith DM, Glasgow SM, Hori K, Dumas J, MacDonald RJ, Johnson JE (2009) Neurog2 is a direct downstream target of the Ptfla-Rbpj transcription complex in dorsal spinal cord. Development 136:2945-2954.

Imayoshi I, Sakamoto M, Yamaguchi M, Mori K, Kageyama R (2010) Essential roles of Notch signaling in maintenance of neural stem cells in developing and adult brains. J Neurosci 30:3489-3498.

Jadhav AP, Mason HA, Cepko CL (2006) Notch 1 inhibits photoreceptor production in the developing mammalian retina. Development 133:913-923.

Kageyama R, Ohtsuka T, Shimojo H, Imayoshi I (2008) Dynamic Notch 
signaling in neural progenitor cells and a revised view of lateral inhibition. Nat Neurosci 11:1247-1251.

Kan L, Israsena N, Zhang Z, Hu M, Zhao LR, Jalali A, Sahni V, Kessler JA (2004) Sox 1 acts through multiple independent pathways to promote neurogenesis. Dev Biol 269:580-594.

Kiernan AE, Cordes R, Kopan R, Gossler A, Gridley T (2005a) The Notch ligands DLL1 and JAG2 act synergistically to regulate hair cell development in the mammalian inner ear. Development 132:4353-4362.

Kiernan AE, Pelling AL, Leung KK, Tang AS, Bell DM, Tease C, Lovell-Badge $\mathrm{R}$, Steel KP, Cheah KS (2005b) Sox2 is required for sensory organ development in the mammalian inner ear. Nature 434:1031-1035.

Lanford PJ, Lan Y, Jiang R, Lindsell C, Weinmaster G, Gridley T, Kelley MW (1999) Notch signalling pathway mediates hair cell development in mammalian cochlea. Nat Genet 21:289-292.

Lewis AK, Frantz GD, Carpenter DA, de Sauvage FJ, Gao WQ (1998) Distinct expression patterns of notch family receptors and ligands during development of the mammalian inner ear. Mech Dev 78:159-163.

Li H, Liu H, Heller S (2003a) Pluripotent stem cells from the adult mouse inner ear. Nat Med 9:1293-1299.

Li H, Roblin G, Liu H, Heller S (2003b) Generation of hair cells by stepwise differentiation of embryonic stem cells. Proc Natl Acad Sci U S A 100: 13495-13500.

Lo L, Dormand E, Greenwood A, Anderson DJ (2002) Comparison of the generic neuronal differentiation and neuron subtype specification functions of mammalian achaete-scute and atonal homologs in cultured neural progenitor cells. Development 129:1553-1567.

Lumpkin EA, Collisson T, Parab P, Omer-Abdalla A, Haeberle H, Chen P, Doetzlhofer A, White P, Groves A, Segil N, Johnson JE (2003) Math1driven GFP expression in the developing nervous system of transgenic mice. Gene Expr Patterns 3:389-395.

Martinez-Monedero R, Yi E, Oshima K, Glowatzki E, Edge AS (2008) Differentiation of inner ear stem cells to functional sensory neurons. Dev Neurobiol 68:669-684.

Matei V, Pauley S, Kaing S, Rowitch D, Beisel KW, Morris K, Feng F, Jones K, Lee J, Fritzsch B (2005) Smaller inner ear sensory epithelia in Neurog 1 null mice are related to earlier hair cell cycle exit. Dev Dyn 234:633-650.

Murray RC, Tapscott SJ, Petersen JW, Calof AL, McCormick MB (2000) A fragment of the Neurogenin 1 gene confers regulated expression of a reporter gene in vitro and in vivo. Dev Dyn 218:189-194.

Neves J, Kamaid A, Alsina B, Giraldez F (2007) Differential expression of Sox 2 and Sox 3 in neuronal and sensory progenitors of the developing inner ear of the chick. J Comp Neurol 503:487-500.

Ohyama T, Mohamed OA, Taketo MM, Dufort D, Groves AK (2006) Wnt signals mediate a fate decision between otic placode and epidermis. Development 133:865-875.

Oshima K, Grimm CM, Corrales CE, Senn P, Martinez Monedero R, Géléoc GS, Edge A, Holt JR, Heller S (2007) Differential distribution of stem cells in the auditory and vestibular organs of the inner ear. J Assoc Res Otolaryngol 8:18-31.

Puligilla C, Dabdoub A, Brenowitz SD, Kelley MW (2010) Sox2 induces neuronal formation in the developing mammalian cochlea. J Neurosci 30:714-722.

Raft S, Koundakjian EJ, Quinones H, Jayasena CS, Goodrich LV, Johnson JE, Segil N, Groves AK (2007) Cross-regulation of Ngn1 and Math1 coordinates the production of neurons and sensory hair cells during inner ear development. Development 134:4405-4415.

Rubel EW, Fritzsch B (2002) Auditory system development: primary auditory neurons and their targets. Annu Rev Neurosci 25:51-101.

Satoh T, Fekete DM (2005) Clonal analysis of the relationships between mechanosensory cells and the neurons that innervate them in the chicken ear. Development 132:1687-1697.

Savary E, Sabourin JC, Santo J, Hugnot JP, Chabbert C, Van De Water T, Uziel A, Zine A (2008) Cochlear stem/progenitor cells from a postnatal cochlea respond to Jagged 1 and demonstrate that notch signaling promotes sphere formation and sensory potential. Mech Dev 125:674-686.

Shen Q, Wang Y, Dimos JT, Fasano CA, Phoenix TN, Lemischka IR, Ivanova NB, Stifani S, Morrisey EE, Temple S (2006) The timing of cortical neurogenesis is encoded within lineages of individual progenitor cells. Nat Neurosci 9:743-751.

Stone JS, Cotanche DA (2007) Hair cell regeneration in the avian auditory epithelium. Int J Dev Biol 51:633-647.

Van Raay TJ, Moore KB, Iordanova I, Steele M, Jamrich M, Harris WA, Vetter ML (2005) Frizzled 5 signaling governs the neural potential of progenitors in the developing Xenopus retina. Neuron 46:23-36.

Wheeler SR, Stagg SB, Crews ST (2008) Multiple Notch signaling events control Drosophila CNS midline neurogenesis, gliogenesis and neuronal identity. Development 135:3071-3079.

Woods C, Montcouquiol M, Kelley MW (2004) Math1 regulates development of the sensory epithelium in the mammalian cochlea. Nat Neurosci $7: 1310-1318$

Yamamoto N, Tanigaki K, Tsuji M, Yabe D, Ito J, Honjo T (2006) Inhibition of Notch/RBP-J signaling induces hair cell formation in neonate mouse cochleas. J Mol Med 84:37-45.

Yaron O, Farhy C, Marquardt T, Applebury M, Ashery-Padan R (2006) Notch 1 functions to suppress cone-photoreceptor fate specification in the developing mouse retina. Development 133:1367-1378.

Zhang T, Tran S, Clouser C, Pignoni F (2005) Nicastrin controls aspects of photoreceptor neuron specification and differentiation in the Drosophila eye. Dev Dyn 234:590-601.

Zine A, Aubert A, Qiu J, Therianos S, Guillemot F, Kageyama R, de Ribaupierre F (2001) Hes1 and Hes5 activities are required for the normal development of the hair cells in the mammalian inner ear. J Neurosci 21: $4712-4720$. 